\title{
COVERING THEOREMS*
}

BY R. L. MOORE

A set of segments will be said to cover a point set $F$ in the Vitali sense if, for every point $P$ which belongs to $F$ and every positive number $e$, there exists a segment of $G$ which contains $P$ and is of length less than $e$. J.Splawa-Neyman has shown $\dagger$ that if, in space of one dimension, $F$ is a closed and bounded point set of measure zero and $G$ is a set of segments which covers $F$ in the Vitali sense, then, for every positive number $e$, the set $G$ contains a subset $G_{e}$ such that $G_{e}$ covers $F$ and such that the sum of the lengths of the segments of the set $G_{\theta}$ is less than $e$. He cites the question, raised by Sierpinski, whether this theorem remains true after the removal of the condition that $F$ be closed. I have recently $\ddagger$ answered this question in the negative. Splawa-Neyman shows, by an example, that his theorem does not hold true for two dimensions, but makes the following statement, without proof:

"Remarquons que notre théorème subsiste pour les espaces à $n$ dimensions, s'il existe pour tout point $p$ de $F$ une sphére appartenant à $F$ de rayon aussi petit que l'on veut et dont le centre est en p."

In the present paper I will show that the theorem thus stated, without proof, by Splawa-Neyman remains true on the removal of the condition that $F$ be closed. I will also show that the condition that each point of $F$ be the center of spheres of

* Presented to the Society, in a somewhat different form, December 30, 1924. In the abstract of this paper printed in this BuLLETIN, vol. 31 (1925), pp. 219-220, proposition (2) is not correctly worded and, as will be shown below, (3) is false.

$\dagger$ Sur un théorème métrique concernant les ensembles fermés, Fundamenta Mathematicae, vol. 5 (1924), pp. 328-330.

$\ddagger$ Cf. R. L. Moore, Concerning sets of segments which cover a point set in the Vitali sense, Proceedings of the National ACAdemy, vol. 10 (1924), pp. 464-467. 
$G$ of arbitrarily small radius may be replaced by a weaker condition. These results will be established with the aid of certain theorems* which may, perhaps, be of some interest in themselves. I will first consider these theorems.

THEOREM 1. If $0<k<1, e<0$, and $G$ is a set of circular regions $\dagger$ all of radius greater than $e$ and, for every region $g$ of the set $G$, $h_{g}$ is a circular region, concentric with $g$ and with a radius equal to $k$ times the radius of $g$ and $H$ is the set of all such regions $h_{o}$ for all regions $g$ of the set $G$ and the sum of all the regions of the set $G$ is a bounded point set, then the set $G$ contains a finite subset $\bar{G}$ such that every region of the set $H$ lies wholly in some region of the set $\bar{G}$.

Proof. Let $K$ denote the point set composed of the centers of the regions of the set $G$. Let $T$ denote the set of all circular regions $t$, of radius equal to $(1-k) e / 4$, such that $t$ is concentric with some region of the set $H$. It is easy to see that the closed point set $K+K^{\prime}$, composed of $K$ plus its derived set, is covered by $T$. It follows, by the Borel-Lebesgue theorem, that $T$ contains a finite subset $\bar{T}$ which covers $K+K^{\prime}$. For each region $t$ of the set $\bar{T}$ select just one region of $G$ whose center is in $t$ and whose radius is greater than $r_{t}-(1-k) e / 2$, where $r_{t}$ is the least upper bound of the radii of all those regions of $G$ whose centers are in $t$. Let $\bar{G}$ denote the set of all regions so selected. It is easy to see that every region of the set $H$ lies wholly in some region of set $\bar{G}$.

Theorem 2. If $k$ is a positive number less than unity and $G$ is a set of circles and there exists no positive number such that infinitely many circles of the set $G$ have radii greater than that number and, for each circle $g$ of the set $G, h_{g}$ is a circle concentric with $g$ and with a radius equal to $k$ times that of $g$, and $H$ is the totality of all such circles $h_{g}$ for all circles $g$ of the set $G$, then there exists a subset $\bar{G}$ of the set of circles $G$ such that (a) each circle of the set $H$ is within some circle of the set $\bar{G}$, but (b) no circle $\bar{g}$ of

* These theorems will be stated in the terminology of space of two dimensions. It is easy to see however that this restriction is not necessary.

$\dagger$ By a circular region is meant the interior of a circle. 
the set $\bar{G}$ completely encloses any circle $h$, of the set $H$, such that $h=h_{g}$ for some circle $g$, other than $\bar{g}$, of the set $\bar{G}$.

Proof. If for any circle $g$ of the set $G$ there exists another circle of $G$ which is concentric with it let $k_{g}$ denote the largest circle of $G$ which is concentric with $g$ and let $K$ denote the set of all such circles $k_{g}$ for all circles $g$ of the set $G$. Let $\bar{H}$ denote the set of all circles $h$ of the set $H$ such that $h=h_{g}$ for some circle $g$ which belongs to $K$. Clearly every circle of $H$ either is identical with some circle of $\bar{H}$ or is within, and concentric with, some circle of $\bar{H}$. No two circles of $\bar{H}$ are concentric with each other. It follows from the hypothesis that there exists a circle $g_{1}$ which belongs to the set $K$ and which has as large a radius as any other circle of the set $K$. Let $G_{1}$ denote the set of circles consisting of $g_{1}$ together with every circle of $K$ which is not concentric with any circle of $\bar{H}$ which lies within $g_{1}$. No circle of $G_{1}$, except $g_{1}$, encloses $h_{g}$ and $g_{1}$ does not enclose any circle of the set $\bar{H}$ (except $h_{\theta_{1}}$ ) which is concentric with a circle of the set $G_{1}$. Furthermore every circle of the set $H$ is within some circle of the set $G_{1}$. If $G_{1}$ contains any circle other than $g_{1}$ there exists, in the set $G_{1}$, a circle $g_{2}$, distinct from $g_{1}$ and with a radius as great as that of any other circle in $G_{1}$ except $g_{1}$. Let $G_{2}$ denote the set of circles consisting of $g_{2}$ together with every circle of the set $G_{1}$ which is not concentric with any circle of the set $\bar{H}$ which lies within $g_{2}$. Clearly $g_{1}$ belongs to $G_{2}$. Continue this process thus obtaining a finite or countably infinite sequence of circles $g_{1}, g_{2}, g_{3}$, . . . and a corresponding sequence of sets of circles $G_{1}, G_{2}, G_{3}$, . . . such that, if $n>1$, $G_{n}$ consists of $g_{n}$ together with every circle of the set $G_{n-1}$ which is not concentric with any circle of the set $\bar{H}$ which lies within $g_{n}$ and if $G_{n}$ contains any circle other than $g_{1}, g_{2}, g_{3}$, $\ldots g_{g}, g_{n}$ then $g_{n+1}$ is a circle belonging to $G_{n}$ and distinct from the circles $g_{1}, g_{2}, g_{3}, \cdots, g_{n}$ and with a radius as great as that of any other circle in $G_{n}$ except $g_{1}, g_{2}, g_{3}, \cdots, g_{n}$, but if $G_{n}$ contains no circle other than $g_{1}, g_{2}, g_{3}, \cdots, g_{n}$ (i.e. if every circle belonging to $G_{n-1}$, except $g_{1}, g_{2}, g_{3}, \cdots, g_{n}$, is concentric with some circle of the set $\bar{H}$ which lies within $g_{n}$ ) 
then the sequences $g_{1}, g_{2}, g_{3}, \cdot$ • . and $G_{1}, G_{2}, G_{3}$, • • · are finite and $g_{n}$ and $G_{n}$ are their last terms. The finite or countably infinite set of circles $g_{1}, g_{2}, g_{3}$, . . . satisfies all the requirements indicated for the set $\bar{G}$.

THEOREM 3. If $k_{1}$ and $k_{2}$ are positive numbers $\left(1>k_{2}>k_{1}\right)$ and $D$ is a bounded domain and $G$ is a set of circular regions lying in $D$ and, for every region of the set $G, h_{0}$ is a circular region concentric with $g$ and with a radius $k_{1}$ times that of $g$ and $H$ is the set of all regions $h_{0}$ for all regions $g$ of the set $G$, then there exists a subset $\bar{G}$ of the set $G$ such that every region of the set $H$ is contained in some region of the set $\bar{G}$ and such that the sum of the areas of all the regions of the set $\bar{G}$ is less than or equal to $4 A /\left(1-k_{2}\right)^{2}$, where $A$ is the area of the domain $D$.

Proof. For each circle $g$ of the set $G$ construct a circular region $t_{g}$ concentric with $g$ and with a radius equal to $k_{2}$ times that of $g$. Let $T$ denote the set of all the regions $t_{g}$ for all regions $g$ of the set $G$. Let $H_{1}$ denote the set of all those regions of the set $H$ whose radii are greater than or equal to 1 . For each positive integer $n$, greater than 1 , let $H_{n}$ denote the set of all those regions of the set $H$ whose radii are greater than or equal to $1 / n$ but less than $1 /(n-1)$. By Theorem 1 , for each $n$, there exists a finite subset $T_{n}$ of the set of regions $T$ such that every region of the set $H_{n}$ is a subset of some region of the set $T_{n}$. Let $\bar{T}$ denote the set of regions composed of all the regions of all the sets $T_{1}, T_{2}, T_{3}, \ldots$. . There exists no positive number such that infinitely many regions of the set $\bar{T}$ have radii greater than that number. It follows, by Theorem 2 , that there exists a subset $\bar{G}$ of the set of regions $G$ such that (a) every region of the set $\bar{T}$ is a subset of some region of the set $\bar{G}$, (b) no region $\bar{g}$ of the set $\bar{G}$ contains any region $t$ of the set $\bar{T}$ such that $t=t_{\theta}$ for some region $g$, other than $\bar{g}$, of the set $\bar{G}$. For each region $g$ of the set $\bar{G}$ let $q_{g}$ denote a circular region concentric with $g$ and having a radius equal to the radius of $g$ multiplied by $\left(1-k_{2}\right) / 2$. Let $Q$ denote the set of all such regions $q_{g}$ for all regions $g$ of the set $\bar{G}$. It is easy to see that no two regions of the set $Q$ have a point in common. Hence the 
sum of the areas of all the regions of this set is less than or equal to $A$. It follows that the sum of the areas of all the regions of the set $\bar{G}$ is less than or equal to $4 A /\left(1-k_{2}\right)^{2}$. But every region of the set $H$ is a subset of some region of the set $\bar{T}$ and therefore of some region of the set $\bar{G}$. The truth of Theorem 3 is therefore established.

THEOREM 4. If $M$ is a bounded point set of measure zero and $k$ is a positive number less than 1 and $G$ is a set of circular regions and for every point $P$ of $M$ and every positive number e there exists, in the set $G$, a region $g$ of radius less than e and such that the distance from $P$ to the center of $g$ is less than $k$ times the radius of $g$, then, for every positive number e, there exists a subset $\bar{G}$ of $G$ such that $\bar{G}$ covers $M$ and such that the sum of the areas of the regions of the set $\bar{G}$ is less than $e$.

PRoof. For each region $g$ of the set $G$ let $h_{g}$ denote a circular region concentric with $g$ and with a radius $k$ times that of $g$. Let $H$ denote the set of all such regions $h_{g}$. Let $\bar{k}$ denote some definite number between 1 and $k$. Suppose $e$ is a positive number. Since $M$ is of measure zero there exists a set $S$ of circular regions covering $M$ and such that the sum of the areas of the regions of $S$ is less than $e(1-\bar{k})^{2} / 4$. For each point $P$ of $M$ there exists a circular region $g$ belonging to the set $G$ and lying in some region of $S$ and such that $P$ lies in the region $h_{g}$. It follows by Theorem 3 that there exists a subset $\bar{G}$ of $G$ such that each region of $H$ is a subset of some region of $\bar{G}$, and the sum of the areas of the regions of the set $\bar{G}$ is less than or equal to $4 B /(1-\bar{k})^{2}$, where $B$ is the sum of the areas of the regions of the set $S$. Since $B$ is less than $e(1-k)^{2} / 4$ it follows that the sum of the areas of the regions of the set $\bar{G}$ is less than $e$.

Definitions. By a simple chain of segments in space of one dimension is meant a set $C$ of segments such that (a) the point set obtained by adding together the points of all the segments of $C$ is connected, (b) there exists no point which is common to more than two segments of $C$, and (c) no segment of $C$ is a subset of any other segment of $C$. 
By a simple chain of intervals in space of one dimension is meant a set $C$ of intervals such that (a) the point set obtained by adding together the points of all the intervals of $C$ is connected, (b) there exists no point which is common to more than two intervals of the set $C$, (c) no interval of $C$ is a part of any other interval of $C$, and (d) no endpoint of an interval of $C$ is a limit point of a set of endpoints of intervals of $C$.

THEOREM 5. If, in space of one dimension, $G$ is a set of $\left\{\begin{array}{l}\text { segments } \\ \text { intervals }\end{array}\right\}$ and there exists no positive number such that infinitely many $\left\{\begin{array}{l}\text { segments } \\ \text { intervals }\end{array}\right\}$ of the set $G$ are of length greater than that number then $G$ contains a subset $\bar{G}$ such that (a) every point that belongs to $\left\{\begin{array}{l}\text { a segment } \\ \text { an interval }\end{array}\right\}$ of $G$ belongs also to some $\left\{\begin{array}{l}\text { segment } \\ \text { interval }\end{array}\right\}$ of $\bar{G}$, (b) $G$ consists of a set of nonoverlapping simple chains of $\left\{\begin{array}{l}\text { segments. } \\ \text { intervals. }\end{array}\right\}$

The truth of Theorem 5, for the case where $G$ is a set of segments, is established in the course of the argument given to prove Theorem 1 of my Procendings paper referred to above. For the case where $G$ is a set of intervals the same argument will apply if the word "segment" is replaced, at times by the word "interval" and at times by the phrase "other interval."

Theorem 6. If, in space of one dimension, $K$ is a bounded point set of measure zero and $G$ is a set of intervals such that, for each positive number e, each point of $K$ belongs to at least one interval of $G$ of length less than e and there exists no positive number such that infinitely many intervals of the set $G$ are of length greater than that number, then, for every positive number $e, G$ contains a finite, or countably infinite, subset $G_{e}$ such that $G_{e}$ covers $K$ and such that the sum of the lengths of the intervals of $G_{e}$ is less than $e$ and such that, furthermore, $G_{e}$ consists of a countable number of nonoverlapping simple chains of intervals.

With the help of Theorem 5, Theorem 6 may be proved by an argument similar to that used to prove Theorem 1 of the above mentioned paper. 
Splawa-Neyman indicates that his theorem becomes true for a point set $F$ of measure greater than zero if the condition that the sum of the lengths of the segments of the set $G_{e}$ be less than $e$ is replaced by the condition that it be less than $m(F)+e$, where $m(F)$ is the measure of $F$. Theorems 4 and 6 of the present paper may be generalized in an analogous manner. The truth of this statement for the case of Theorem 6 may be seen with the help of the fact that, under the hypothesis of this theorem (modified by the omission of the requirement that $K$ be of measure zero), the set $G$ contains as a subset a set $S$ of nonoverlapping intervals such that (a) every interval of $S$ lies in a certain predetermined domain whose measure exceeds the measure of $F$ by less than $e / 2$, (b) $S$ covers the whole of $F$ except for a point set of measure zero. The existence of such a set $S$ follows from Carathéodory's generalization* of Vitali's theorem. A largely similar argument holds good for the case of Theorem 4 .

That Splawa-Neyman's theorem does not remain true if the words "à son intérieur" are omitted may be seen as follows. Using the notation of the example given on pages 464 and 465 of my PROCEEdings paper, for each positive integer $n$ let $K_{n}$ denote the point set consisting of the left ends of all the segments of the set $G_{n}$. For each point $P$ of $K_{n}$ let $I_{P}$ denote the smallest segment of $G_{n}$ which has $P$ as its left end and let $H_{P}$ denote a set of intervals having $P$ as their common left end, their right ends forming an infinite sequence of points $A_{P 1}$, $A_{P 2}, A_{P 3}, \cdot \cdots \cdot$ such that, for every positive integer $j$, the length of the interval whose end points are $P$ and $A_{P_{j}}$ is equal to the length of $I_{P}$ divided by $2 j$. For each $n$ let $Q_{n}$ denote the set of intervals composed of all the intervals of all the sets $H_{P}$ for all points $P$ of $K_{n}$. Let $Q$ denote the set of intervals composed of all the intervals of all the sets $Q_{1}, Q_{2}, Q_{3}, \cdot$. . , together with a set of intervals having $B$ as their common left end, their right ends forming an infinite sequence of points $B_{1}, B_{2}, B_{3}, \cdots \cdot$, all lying to the right of $B$ and such that the

* Cf. C. Carathéodory, Vorlesungen über reelle Funktionen, Teubner, 1918, p. 304. 
distance from $B_{j}$ to $B$ is equal to $1 / j$. Let $W$ denote the set of intervals composed of all the intervals of $Q$ and all intervals $t$ such that $t$ is equal to some segment of $G$ plus its endpoints. Let $F$ denote the point set $B+K+K_{1}+K_{2}+K_{3}+$. . . . It can be seen that $F$ is a closed point set of measure zero and that it is covered in the Vitali sense by the set of intervals $W$. But $W$ contains no subset $\bar{W}$ such that $\bar{W}$ covers $F$ and such that the sum of the lengths of the intervals of $\bar{W}$ exists (as a finite number).

The University of Texas

\title{
THE CONVERGENCE OF A GENERAL MEAN OF MEASUREMENTS TO THE TRUE VALUE*
}

\author{
BY E. L. DODD
}

Theorem. For positive measurements $x_{i}$ suppose that the frequency function $\phi(x)$ has a positive lower bound $b$ in some interval $(a-k, a+k)$ about the true value $a$, and that for each positive $r$

$$
m_{r}=\int_{0}^{\infty} x^{r} \phi(x) d x, \quad(\text { finite }) ; \quad m_{0}=1 .
$$

Then there exists a continuous increasing function $f(x)$, with inverse $f^{-1}$, such that if $\epsilon>0, \eta>0$, and

(2) $M=f^{-1}\left\{\right.$ Arithmetic mean of $\left.f\left(x_{i}\right)\right\}, i=1,2, \cdots, n$, then, when $n>$ some $n^{\prime}$, there is a probability $>1-\eta$ that

$$
|M-a|<\epsilon \text {. }
$$

Proof. For (3) the special condition $\dagger$ is

$$
f(a)=\int_{0}^{\infty} f(x) \phi(x) d x .
$$

* Presented to the Society, February 27, 1926.

$\dagger$ Dodd, Functions of measurements under general laws of error, SkandNavisk AkTUaRietidskRift, vol. 5 (1922), pp. 133-158. Apply theorem, p. 135, taking $\Sigma f\left(x_{i}\right)<n f(a+\epsilon)=n(1+\delta) f(a)=z$; then $\Sigma f\left(x_{i}\right)<n f(a-\epsilon)$. 